\title{
Measuring tidal volume and functional residual capacity change in sleeping infants using a volume displacement plethysmograph
}

\author{
S.J. Wilson*, C. O'Brien+, M.A. Harris' ${ }^{+}$I.B. Masters ${ }^{+}$
}

Measuring tidal volume and functional residual capacity change in sleeping infants using a volume displacement plethysmograph. S.J. Wilson, C. O'Brien, M.A. Harris, I.B. Masters. OERS Journals Ltd 1998.

ABSTRACT: The noninvasive measurement of infant lung function during unsedated sleep in infants has been a long-standing objective in paediatric respiratory medicine. This note reports on the design and performance of a head-out volume-displacement plethysmograph (VDP) that overcomes some of the limitations of traditional lung function apparatus.

The VDP comprises a rigid acrylic box with an integral water-sealed spirometer and a novel neck seal. The bilayer neck seal is of variable compliance and is comfortable and simple to use. The spirometer permits volume resolution of $1.5 \mathrm{~mL}$ and a dynamic range in excess of $100 \mathrm{~mL}$. The frequency response extends from $0-7 \mathrm{~Hz}$. Spirometer inertance was measured as $0.0015 \mathrm{kPa} \cdot \mathrm{L}^{-1} \cdot \mathrm{s}^{-2}$, resistance $0.021 \mathrm{kPa} \cdot \mathrm{L}^{-1} \cdot \mathrm{s}^{-1}$ and box capacitance $0.18 \mathrm{~L} \cdot \mathrm{kPa}^{-1}$.

Tidal volume, respiratory rate and changes in functional residual capacity can be recorded during unsedated rapid eye movement and nonrapid eye movement whilst monitoring with conventional polysomnographic methods. The head-out configuration allows additional instrumentation to be implemented with ease, avoids facial stimulation and allows unimpeded access to the upper airway. A polysomnograph illustrating the limitations of respiratory inductance plethysmography signals and typical changes in functional residual capacity are shown.

Eur Respir J 1998; 12: 1186-1190.
*Center for Magnetic Resonance, The University of Queensland, St Lucia, Brisbane, Australia. ${ }^{+}$Dept of Respiratory Medicine, The Mater Children's Hospital, South Brisbane, Australia.

Correspondence: I.B. Masters

Dept of Respiratory Medicine

Mater Misericordiae Children's Hospital South Brisbane

Queensland, 4101

Australia

Fax: 61738408366

Keywords: Functional residual capacity infant-newborn

lung volume measurement

sleep

volume displacement plethysmograph

Received: January 161998

Accepted after revision July 71998

Supported by ResMed Ltd
Objective assessment of respiratory function plays an important part in understanding the physiology and pathophysiology of the respiratory system. Lung function is an indispensable tool for diagnosing and monitoring respiratory disease states in older children, but has not gained wide acceptance in the management of infant lung disease. The primary difficulty in measuring infant lung function is the inherent lack of cooperation, requiring assessment during sedated sleep and the use of a face mask. To date, there is no widely applied technique available for measuring infant lung function in the unsedated infant.

Traditional methods of volume and flow measurement with a face mask and pneumotachograph induce error through the effects of trigeminal stimulation, increased dead space and resistive loading $[1,2]$. Their use is poorly tolerated in unsedated infants, inducing arousal, especially in light sleep, and are completely impractical in the awake infant. In addition, it is technically difficult to maintain a seal with the infant for protracted periods, thus limiting the ability to acquire data dynamically during unpredictable respiratory events such as apnoeas, sighs and hypopnoeas. These events are typically associated with desaturation, and understanding the respiratory dynamics that surround such events forms an important part of sleep medicine.

Respiratory inductance plethysmography (RIP) has been used as a noninvasive measure of tidal volume and minute volume in infants, but is compromised by complex and time-consuming calibration techniques [3-6], although recently a simplified calibration has been described [7].

A pneumotachograph and face mask are still required to calibrate RIP to measure volume accurately and, therefore, can only be used in very young or sedated infants. No studies have validated calibrated RIP in infants outside the neonatal period, although it has been used in cooperative children [8]. A fundamental problem with RIP is the approximation of the infant respiratory system as a twocompartment model. In disease states, chest wall motion is complex, with subcostal and suprasternal recession being typical features. CARLO et al. [9] described chest wall recession in the inferior aspect of the chest with expansion in the upper portion of the chest in infants with respiratory distress syndrome; it is unlikely that a single RIP band can accurately measure changes in thoracic volume in such situations. There have been no studies validating RIP as a measure of tidal volume in infants with lung disease and it is known to be inaccurate in infants under $1.5 \mathrm{~kg}$, presumably because of variable chest wall compliance [10].

Functional residual capacity (FRC) in infants is dynamically elevated above relaxed end expiratory volume using laryngeal and diaphragmatic braking, together with the commencement of inspiration before end-expiration [1114]. The mechanism of FRC elevation, therefore, revolves around an interaction between the expiratory time constant and rate. FRC is usually reported as a single measurement 
and yet, because of the way this volume is achieved by the infant, it is likely that a range of volumes more accurately describes FRC. This is probably the case in rapid eye movement (REM) sleep, which is characterized by a highly variable rate [15] and varying degrees of laryngeal adductor activity [16-18].

FRC change with different sleep states has been explored by a number of groups with conflicting results [1923]. Only STокеS et al. [23] explored the concept of FRC variability using the respiratory jacket. This technique has not gained wide acceptance, presumably owing to difficulties with calibration and fitting of the jacket. In order to interpret FRC measurements and indeed lung mechanics, which are very dependent on lung volume, it is clearly important to understand the natural degree of variability in FRC.

The ability to measure infant lung function noninvasively during unsedated sleep without the need for a face mask would be a significant advance in paediatric respiratory medicine. The aim of this paper was to describe a technique for recording tidal volume and changes and variability in FRC using a volume-displacement head-out plethysmograph during unsedated sleep. This technique allows access to the airway, avoids facial stimulation and facilitates additional monitoring with electroencephalography (EEG), electro-occulography (EOG) and oesophageal manometry. This work is part of a larger project to define the degree of FRC instability with different sleep states in normal infants and infants with lung disease during the first 3 months of life during unsedated sleep.

\section{Methods}

The head-out volume-displacement plethysmograph (VDP) comprises three integral parts, as shown in figure 1 and described below.

\section{Plethysmograph}

A rigid, clear acrylic box was constructed to internal dimensions of $180 \times 320 \times 500 \mathrm{~mm}$ to accommodate the range of infants studied. A removable top plate served as the base of a water-sealed spirometer.

Airtight electrical connections allowed the passage of cabling into the box for electrocardiography (ECG), oximetry and RIP. A port at the side allowed attachment of a

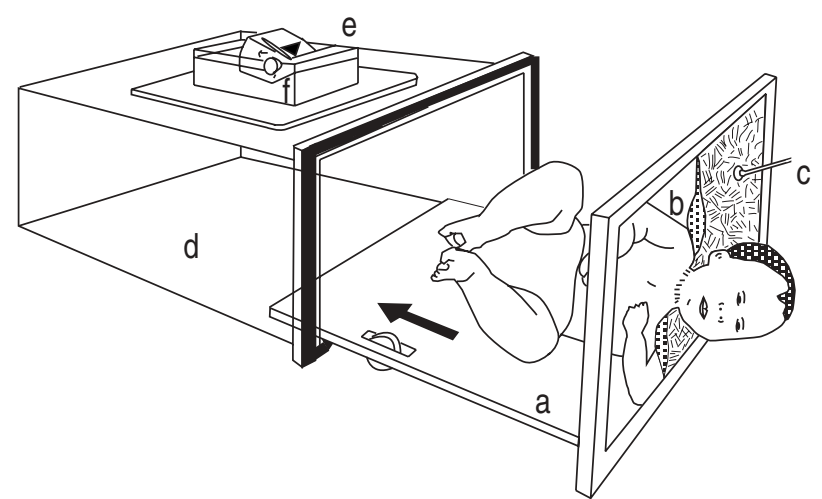

Fig. 1. - Schematic view of the volume-displacement plethysmograph (VDP) showing the principal components and their relationship to the infant. a) Tray; b) bilayered neck seal; c) vacuum port; d) acrylic box; e) spirometer; and f) port to spirometer. calibrated gas syringe for calibration of the spirometer. A mattress tray allowed easy access to the infant to aid settling and facilitated insertion of the infant within the device without disturbance. The neck seal frame was attached to the mattress tray and apposed the entrance of the box when the infant was inserted into the plethysmograph. An airtight seal was achieved with a peripheral gasket. A circulating fan provided air mixing within the chamber through a silica-gel cartridge and ensured that recorded box humidity was controlled in the presence of skin losses. Box temperature and humidity were recorded with battery-operated instruments within the chamber. Multichannel signal acquisition and storage was performed by a Macintosh IIci personal computer running MacLab ${ }^{\circledR}$ hardware and A/D software (Version 3.5.2 AD Instruments, NSW, Australia).

\section{Spirometer}

A modified Krogh-style spirometer [24] with one degree of freedom (radial) was employed rather than a pneumotachograph, to measure volume displacements from the box, because of the superior low-frequency response, extending to $0 \mathrm{~Hz}$. A lightweight polystyrene bell (volume $120 \mathrm{~mL}$, mass $16 \mathrm{~g}$ ) was mounted on the radial arms of a carbon fibre shaft which rotated a linear rotary potentiometer. A port was located at the level of the upper abdomen when the infant was in the box.

\section{Neck seal}

An airtight neck seal is achieved with a bilayer of latex rubber sheet (170 $\mu \mathrm{m}$ mean thickness). Holes of varying sizes from 30-55 mm diameter were cut to accommodate the range of neck circumferences in the infants studied. The space between the latex sheets was filled with expanded polystyrene particles and a rigid vacuum port attached to the outer sheet. With no vacuum applied the structure was compliant and easily passed over the head of the infant. The application of a vacuum $(-10--20 \mathrm{kPa})$ transformed the structure to a rigid membrane whilst still providing a comfortable airtight seal to the neck.

\section{Testing the characteristics of the VDP}

The frequency response and phase characteristics of the VDP were tested using a loudspeaker driven by sinusoidal signals of variable frequency through a D.C.-coupled amplifier. Box acoustic capacitance was tested by the rapid and slow injection of $100 \mathrm{~mL}$ of air while the sealed box pressure was recorded (Validyne LVDT transducer 0-10 $\mathrm{kPa}$ ). Spirometer inertance was calculated by the introduction of a varying signal at the spirometer port whilst the pressure was recorded at the point of introduction (Validyne LVDT transducer 0-0.2 kPa).

\section{Procedure for infant study}

The spirometer was calibrated by inserting known volumes of air into the plethysmograph using a calibrated gas 
Table 1. - Measured characteristics of the 30- $L$ volumedisplacement plethysmograph tested with $2 \mathrm{~L}$ normal saline, simulating the infant's volume

\begin{tabular}{ll}
\hline Acoustic capacitance & $0.18 \mathrm{~L} \cdot \mathrm{kPa}^{-1}\left(0.018 \mathrm{~L} \cdot \mathrm{cmH}_{2} \mathrm{O}^{-1}\right)$ \\
Resistance & $0.021 \mathrm{kPa} \cdot \mathrm{L}^{-1} \cdot \mathrm{s}^{-1}\left(0.21 \mathrm{cmH}_{2} \mathrm{O} \cdot \mathrm{L}^{-1} \cdot \mathrm{s}^{-1}\right)$ \\
Inertance & $0.0015 \mathrm{kPa} \cdot \mathrm{L}^{-1} \cdot \mathrm{s}^{-2}\left(0.015 \mathrm{cmH}_{2} \mathrm{O} \cdot \mathrm{L}^{-1} \cdot \mathrm{s}^{-2}\right)$ \\
\hline
\end{tabular}

syringe prior to insertion of the infant. After monitoring devices were applied to the infant a normal feed was given and the settled infant placed on the tray. Most infants were studied supine, but the decubitus position and prone position were also used successfully. The infant's head was delivered through the neck seal aperture, which was stretched open by the operator.

Application of a vacuum then stabilized the seal in an airtight position whilst conforming to the infants sleep position. Any leak at the seal was apparent from a monophasic baseline drift on the spirometer output and data were discarded until the seal was re-established. This involved minor positioning manoeuvres of the infant's head and neck.

The box temperature was elevated $1.5-2^{\circ}$ above the am-bient to a steady state with changes being $<1.0^{\circ} \cdot \mathrm{h}^{-1}$. Gross body movements that induced artefacts on the volume signal were edited accordingly. Written consent was obtained from parents and the study approved by the Mater Children's Hospital Research and Ethics Committee.

\section{Results}

The capacitance, resistance and inertance of the plethysmograph and spirometer are presented in table 1 .

Eight normal infants and seven infants with varying degrees of airway and interstitial lung disease aged from

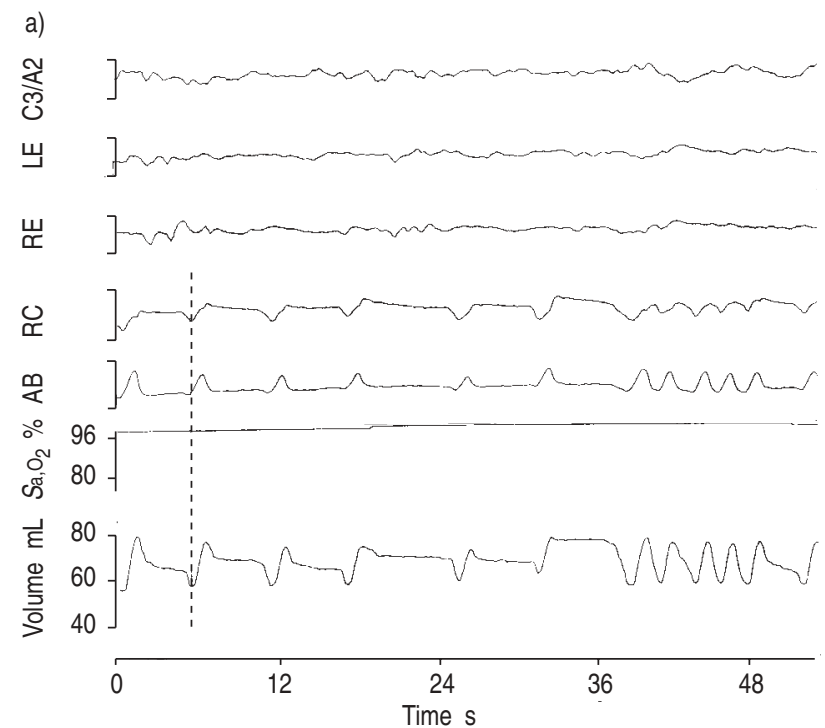

term to 3 months were studied successfully. Some infants were studied for over $2 \mathrm{~h}$, with the limiting variable usually being the timing of the next feed. No complications were encountered and most infants settled spontaneously. Difficulties were experienced with some larger infants who were disturbed by the restrictive space within the plethysmograph. One infant failed to settle in the VDP owing to the restrictive space and the study was abandoned. All infants were fed, prepared and settled in the box within $1 \mathrm{~h}$.

Polysomnographs of the infants studied in the VDP are presented in figure 2 illustrating the ability to record tidal volume, changes in FRC and sigh volume in unsedated sleep.

Figure 2a illustrates how misleading conventional RIP can be in accurately representing tidal breathing. The RIP channels appear on first inspection to be a typical paradox; however, analysis of the volume channel shows that this infant is adopting an exaggerated form of expiratory braking or breath-hold. It can be seen that the paradoxical inward movement of the chest wall is partly due to expiration of the previous breath.

The continuation of ribcage contraction beyond end-expiration reflects the response of the compliant chest wall to the commencement of the next inspiratory effort.

\section{Discussion}

A device has been successfully developed for monitoring tidal volume, respiratory rate and changes in FRC in unsedated sleep in infants with and without lung disease. Data were successfully obtained in REM and non-REM (NREM) sleep. The mechanical properties of the plethysmograph and spirometer are adequate for recording tidal breathing parameters in infants [25]. The spirometer could easily be developed and used in more traditional apparatus displaying adequate frequency response, resolution and linear

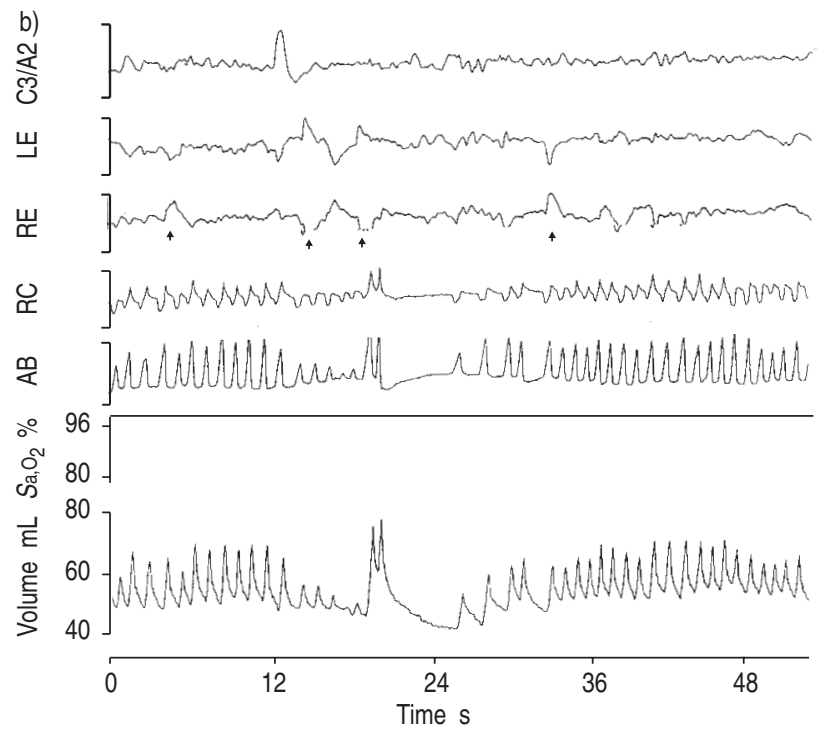

Fig. 2. - Polysomnographic recordings. a) Sample recording of a $2.2 \mathrm{~kg}$ infant of corrected age 36 weeks studied in the volume-displacement plethysmograph (VDP). An exaggerated form of expiratory braking is illustrated and the respiratory inductance plethysmograph (RIP) suggests respiratory paradox. - - - : shows lag in the ribcage (RC) expiration signal relative to actual volume loss. b) Recording of an infant with bronchopulmonary dysplasia at 41.5 weeks corrected age and weight $3.8 \mathrm{~kg}$ in rapid eye movement (REM) sleep showing fluctuations in the baseline of the spirometer signal. This represents variations in functional residual capacity in the order of $20 \mathrm{~mL}$ over the epoch displayed. All records show, from the top down, electroencephalogram (EEG; C3/A2), electro-oculogram (EOG) leads (LE, RE), RC and abdominal (AB) RIP signal, oxygen saturation ( $\mathrm{Sa}$, $\mathrm{O}_{2}$ ) and volumedisplacement signal. Inspiration is up on both RIP and volume signals. $\uparrow$ : denote eye movements. 
dynamic range for monitoring tidal breathing in infants. The differentiated volume signal (flow) would be suitable for the determination of tidal breathing timing indices such as time to peak expiratory flow ( $t \mathrm{PTEF}) /$ expiratory time $(t \mathrm{E})$. The advantages of the spirometer over a pneumotachograph are stability of calibration, reduced resistive load and low-frequency response extending to $0 \mathrm{~Hz}$. The inferior low frequency response of the pneumotachograph is caused by very small pressure drops across the resistive element at low flows, making it less likely to resolve accurately the slow FRC drifts seen in sleep.

The novel neck seal described is comfortable, easy to apply and mechanically stable, and permits natural sleep. Mechanical stability ensures that volume displacements within the box are accurately represented by the spirometer without deformation of the neck seal. Obstruction of cerebral blood flow due to a circumferential latex ring about the neck is a theoretical concern; however, minimal contact pressure is required on the neck of the infant in order to obtain a seal, because of the very small static pressure of the spirometer. PALMER et al. [26] investigated the effects of neck seals, used in negative pressure ventilation, on cerebral circulation. They demonstrated no significant jugular occlusion using a latex seal $350 \mu \mathrm{m}$ in thickness, greater than twice the thickness of the neck seal used here.

This device allows monitoring of FRC changes on a breath-by-breath basis over protracted periods, thus allowing a quantitative measure of FRC instability to be made in infants. The data are unobtainable with the washout/ dilution techniques and constant-volume plethysmography, which tender a static measurement of FRC. These techniques also require the use of a face mask and pneumotachograph, which may alter FRC by the effects of resistive loading on timing indices $[27,28]$, trigeminal stimulation and increased dead space $[1,2]$.

Earlier studies on the accuracy of RIP as a measure of tidal volume are flawed by the use of correlation as an index of accuracy [29]. AdAMs et al. [7] used appropriate statistical techniques and have shown that RIP provides a satisfactory representation of tidal volume, although no attempt was made to describe FRC changes. In order to show changes in FRC over relatively long periods the D.C. coupled mode should be used.

The calibration in this mode is, however, very sensitive to movement and the technique has not yet been used for tracking FRC changes.

The VDP, first brought to prominence by MEAD [30], allows comparatively simple instrumentation to measure accurately tidal and forced breathing parameters in adults. The insignificant compression and rarefaction of box gas occurring during tidal breathing are isothermal in nature [31] and eliminate the requirement for the complex calibration due to polytropic behaviour seen in pressure plethysmographs [32].

Face masks and mouthpieces were implemented for cooperative adult subjects when using the VDP but these are unsuitable for infants. Various face seals for use in infants have been described using a pneumatic cuff [33] or a face cuff [34] applied with downward pressure. Both techniques would result in significant orofacial stimulation and jaw displacement with compromise of the upper airway and are unlikely to be tolerated in unsedated older infants.
In conclusion, a method has been developed for measuring tidal volume and change in functional residual capacity dynamically that avoids the use of a face mask. The device demonstrates mechanical and clinical performance suitable for monitoring sleeping infants for prolonged periods without sedation. The device is simply and quickly calibrated. Furthermore, the interpretation of respiratory phenomena derived from conventional noninvasive monitoring is enhanced. An expanded role is envisaged in the investigation of dynamic respiratory mechanics of the sleeping infant. The head-out architecture will permit various therapeutic interventions to be monitored without orofacial stimulation.

\footnotetext{
Acknowledgement: The authors gratefully acknowledge the assistance of J. Williams in the preparation and supervision of the infants examined.
}

\section{References}

1. Dolfin T, Duffty P, Wilkes D, England S, Bryan H. Effects of a face mask and pneumotachograph on breathing in sleeping infants. Am Rev Respir Dis 1983; 128: 977979.

2. Fleming $\mathrm{P}$, Levine M, Goncalves A. Changes in respiratory pattern resulting from the use of a facemask to record respiration in newborn infants. Pediatr Res 1982; 16: 1031-1034.

3. Dolfin T, Duffty P, Wilkes D, Bryan M. Calibration of respiratory inductance plethysmograph (Respitrace) in infants. Am Rev Respir Dis 1982; 126: 577-579.

4. Chadha T, Watson H, Birch S, Jenouri S. Validation of respiratory inductance plethysmography using different calibration procedures. Am Rev Respir Dis 1982; 125: 644-666.

5. Revow MD, England SJ, Stogryn HA, Wilkes DL. Comparison of calibration methods for respiratory inductive plethysmography in infants. J Appl Physiol 1987; 63: 1853-1861.

6. Stefano JL, Spitzer AR, Baurngart S, Davis JM, Fox WW. Inductive plethysmography - a facilitated postural calibration technique for rapid and accurate tidal volume determination in low birth weight premature newborns. Am Rev Respir Dis 1986; 134: 1020-1024.

7. Adams J, Zabaleta I, Stroh D, Johnson P, Sackner M. Tidal volume measurements in newborn using respiratory inductance plethysmography. Am Rev Respir Dis 1993; 148: 585-588.

8. Tabachnik E, Muller N, Toye B, Levison H. Measurement of ventilation in children using the respiratory inductive plethysmograph. J Pediatr 1981; 99: 895-899.

9. Carlo W, Martin R, Versteegh F, Goldman M, Robertson $\mathrm{S}$, Fanaroff A. The effect of respiratory distress syndrome on chest wall movements and respiratory pauses in preterm infants. Am Rev Respir Dis 1982; 126: 103-107.

10. Wilkes D, Revow M, Bryan M, England S. Evaluation of respiratory inductance plethysmography in infants weighing less than 1500 grams. Am Rev Respir Dis 1987; 136: 416-419.

11. Kosch PC, Stark AR. Dynamic maintenance of end-expiratory lung volume in full-term infants. $J$ Appl Physiol 1984; 57: 1126-1133.

12. Mortola J, Milic-Emili J, Smith B, Fox G, Weeks S. Dynamics of breathing in infants. J Appl Physiol 1982; 52: $1209-1215$. 
13. Kosch PC, Hutchinson AA, Wozniak JA, Carlo WA, Stark AR. Posterior cricoarytenoid and diaphragm activities during tidal breathing in neonates. J Appl Physiol 1988; 64: 1968-1978.

14. Olinsky A, Bryan M, Bryan A. Influence of lung inflation on respiratory control in neonates. J Appl Physiol 1974; 36: 426-429.

15. Haddad G, Lai T, Epstein M, et al. Breath to breath variations in rate and depth of ventilation in sleeping infants. Am J Physiol 1982; 243: R164-R169.

16. Harding R. Sleep related and developmental changes in laryngeal function. Sleep 1980; 3: 307-322.

17. Harding R, Johnson P, McClelland M. Respiratory function of the larynx in developing sheep and the influence of sleep state. Respir Physiol 1980; 40: 165-179.

18. England S, Kent G, Stogyn H. Laryngeal muscle and diaphragmatic activities in conscious pups. Respir Physiol 1985; 60: 95-108.

19. Henderson-Smart DJ, Read WC. Reduced lung volume during behavioural active sleep in newborn. J Appl Physiol 1979; 46: 1081-1085.

20. Moriette G, Chaussain M, Radvanyi Bouvet MF, Walti H, Pajot N, Relier JP. Functional residual capacity and sleep states in the premature newborn. Biol Neonate 1983; 43: $125-133$.

21. Walti H, Moriette G, Radvanyi Bouvet MF, et al. Influence of breathing pattern on functional residual capacity in sleeping newborn infants. J Dev Physiol 1986; 8: 167172.

22. Beardsmore CS, MacFadyen UM, Moosavi SS, Wimpress SP, Thompson J, Simpson H. Measurement of lung volumes during active and quiet sleep in infants. Pediatr Pulmonol 1989; 7: 71-77.

23. Stokes G, Milner A, Newball E, Smith N, Dunn C, Wilson A. Do lung volumes change with sleep state in the neonate? Eur J Pediatr 1989; 148: 360-364.

24. Krogh A, Lindhard J. Measurement of the blood flow through lungs of man. Skand Arch Physiol 1912: 100-124.

25. Bates J, Turner M, Lanteri C, Jonson B, Sly P. Measurement of flow and volume. In: Stocks J, Sly P, Tepper R, Morgan W, eds. Infant Respiratory Function Testing. New York, Wiley-Liss 1996; pp. 81-116.

26. Palmer K, Spencer A, Wickramasinghe $\mathrm{Y}$, Wright $\mathrm{T}$, Samuels M, Rolfe P. Negative extrathoracic pressure ventilation-evaluation of the neck seal. Early Hum Dev 1994; 37: 67-72.

27. Kosch PC, Davenport PW, Wozniak JA, Stark AR. Reflex control of expiratory duration in newborn infants. $J$ Appl Physiol 1985; 58: 575-581.

28. Davenport P, Frazier D, Zechman F. The effect of the resistive loading of inspiration and expiration on pulmonary stretch receptor discharge. Respir Physiol 1981; 43: 299-314.

29. Bland JM, Altman DG. Statistical methods for assessing agreement between two methods of clinical measurement. Lancet 1986; 1: 307-310.

30. Mead J. Volume displacement plethysmograph for respiratory measurements in human subjects. J Appl Physiol 1960; 15: 736-740.

31. Clement J, Woestijne K. Pressure correction in volume and flow displacement body plethysmography. J Appl Physiol 1969; 27: 895-897.

32. Sly P, Lanteri C, Bates J. Effects of the thermodynamics of an infant plethysmograph on the measurement of thoracic gas volume. Pediatr Pulmonol 1990; 8: 203-208.

33. Cook C, Sutherland J, Segal S, Mead C, Mcllroy B, Smith C. Studies of respiratory physiology in the newborn infant. III. Measurements of mechanics of respiration. $J$ Clin Invest 1957; 36: 440-448.

34. Hjalmarson O. Mechanics of breathing in newborn infants with pulmonary disease. II. Theoretical considerations and methods. Acta Pediatr Scand 1974; 247: Suppl., 6-25. 